\title{
Maternal Antibody Improves Active Neonatal Vaccination against Respiratory Syncytial Virus through Immune Complex
}

Zhilong Jiang ${ }^{*}$, Dandan Li and Lei Zhu

Department of Pulmonary Medicine, Zhongshan Hospital, Fudan University, 180 Feng Lin Road, Shanghai, PR China

*Corresponding author: Zhilong Jiang, Department of Pulmonary Medicine, Zhongshan Hospital, Fudan University, 180 Feng Lin Road, Shanghai, PR China, Tel: +862164041990; Fax: +862164035399; E-mail: jiang.zhilong@zs-hospital.sh.cn

Received date: Jul 31, 2016; Accepted date: Aug 22, 2016; Publication date: Aug 26, 2016

Copyright: @ 2016 Jiang Z, et al. This is an open-access article distributed under the terms of the Creative Commons Attribution License, which permits unrestricted use, distribution, and reproduction in any medium, provided the original author and source are credited.

\begin{abstract}
Respiratory syncytial virus (RSV) is a common infectious pathogen in infants. Vaccination is an important approach in the prevention of RSV infection in early age, but early vaccination is limited by low systemic and cellular immune responses. To overcome this difficulty, we in this study explored a novel vaccination strategy in a mouse model. Pregnant mother was vaccinated at day 14 of gestation and offspring were actively vaccinated at day 3 after birth. We observed a high level of maternal anti-RSV antibody in offspring born to the RSV immunized mother, but it declined 6 weeks after birth. However, anti-RSV antibody was low in offspring received active immunization alone. After offspring were nasal challenged with $2.8 \times 10^{5}$ i.u. RSV at age of 6 weeks old, we observed significant low levels of lung RSV load and inflammation in offspring received both passive and active vaccination as compared to the offspring received passive or active vaccination alone. The increased protection was associated with the increased endogenous production of anti-RSV antibody and Th1-biased cytokine IFN-gamma. Further in vitro study confirmed that maternal anti-RSV antibody and RSV antigen formed immune complex. The splenocytes stimulated with RSV immune complex expressed higher level of IFN-gamma than those stimulated with RSV alone. Thus, maternal anti-RSV antibody increased efficacy of active vaccination in neonates as early as 3 days after birth and provided more protection against RSV than passive or active vaccination alone. Formation of RSV immune complex in respiratory mucusal tissues may contribute to the improved Th1-biased immune responses.
\end{abstract}

Keywords: Respiratory syncytial virus (RSV); Maternal antibody; Neonatal vaccination; Immune complex

\section{Introduction}

Respiratory syncytial virus (RSV) is a major cause of severe low respiratory tract infection in infants and elderly that lead over 3 million hospitalizations and around 66000 deaths worldwide each year [1]. In China, around $18.7 \%$ populations are infected with RSV [2]. The high prevalence among these population is caused by the immature immune system in infants $[3,4]$ and impaired immune cells in elderly $[5,6]$. Recent report showed that high population of immune regulatory cells, mesenchymal stromal cells (MSC), myeloid-derived suppressor cells (MDSC) at early age also contribute to the high infection prevalence [3]. In addition, the immature immune system of infants predisposes infants to developing allergic immune responses through inducing Th2 and Th17-biased polarization [1,7]. NK and T cells are recently reported to particiate in the suppressed immune function in neonates possibly through release of IFN-gamma after RSV infection [8].

Unfortunately there are no effective therapeutics against RSV infection so far. Early vaccination is an effectiveand safe approach in the prevention of RSV infection. Recent study indicated that RSV infection can be effectively prevented by passive immunization via infusion of humanized RSV monoclonal antibody [9]. Studies in animal models and human subjects also confirmed that maternal antiRSV antibody can be vertically transfered into neonates via placenta and breastfeeding $[10,11]$. Thus infants can be passively protected by maternal antibody from immunized mother. However the approach is limited by short-term duration of anti-RSV in offspring [12].
Experiment in animal models showed that maternal antibody antiRSV glycoprotein core fragment (Gcf) diminished half at 3 weeks of age born to RSV immunized mother and the level was almost undetectable at 5 weeks of age [13]. Whereas the endogenous immunity induced by active immunization can provide infants with potent and long-lasting immune protection. Thus it is important for infant to achieved adaptive endogenous immunity against RSV through active immunization. However this approach is currently challenged by low immune responses and Th2 biased $\mathrm{T}$ cell polarization after active neonatal vaccination. A variety of approaches has been recently explored to enhance protective Th1 biased immunity in neonates, such as antigens pre-formulated with a variety of adjuvants (Toll-like receptor agonists, CpG oligodeoxynucleotide, polyI:C, innate defense regulator peptide and polyphosphazene, etc). These adjuvants have been confirmed effective in inducing potent immune protection against RSV infection to some extent in animal models [12,14-16].

However these adjuvants failed to suppress Th2 biased immune responses and lung eosinophilia. For example, intranasal immunization with recombinant RSV nuclear protein $\mathrm{N}$ with detoxified E. coli enterotoxin led to higher lung neutrophil and eosinophil infiltration after RSV challenge, though lung viral load was reduced [15]. Therefore it is important to develop long-lasting and Th1-biased immune responses after active vaccination in infants.

Recent studies showed that animals can be well protected from infection by neonatal active vaccination under the presence of maternal antibody $[12,13]$. However, it is still unknown whether maternal antibody can increase Th1 type immune responses and the underlying mechanisms are elusive. In this study, we vaccinated 
pregnant mice at gestation day 14 and newborn offspring at day 3 after birth. We observed a greater increased protection from RSV infection in offspring as compared to passively or actively immunized offspring alone. The results were in association with higher levels of anti-RSV antibody and IFN-gamma expression. Further in vitro study showed that RSV immune complex may contribute to the enhanced Th1biased immune responses through the increased antigen phagocytosis and processing in antigen-presenting cells (APCs).

\section{Methods}

\section{Preparation of respiratory syncytial virus (RSV)}

RSV strain A2 (ATCC) was amplified in Vero cells (ATCC) for 5 days after initial infection (MOI=0.1). Viral particles were concentrated by ultracentrifuge on cesium chloride $(\mathrm{CsCl})$ gradient at $28,000 \mathrm{rpm}$ for $2 \mathrm{hrs}$ and the concentrated RSV was collected from interface and resuspended in PBS. RSV titer was titrated in Vero cells. Vero cells were infected with serial diluted RSV for 24 hours and fixed with $4 \%$ parafamadyhyde for $10 \mathrm{~min}$ and followed by incubation with rabbit anti-RSV (1:500 dilutions) and HRP-conjugated second antibody. The positive cells were visualized under contrast microscope and manually counted. Viral titer was calculated by the formula: positive cells number $\times$ dilution $\div$ added virus volume and titer was presented as infectious units (i.u.) $/ \mathrm{ml}$. We obtained $1.4 \times 10^{7}$ i.u. $/ \mathrm{ml}$ of the concentrated RSV and $3 \times 10^{5}$ i.u./ml RSV in the conditioned media.

\section{RSV vaccination of pregnant mother and neonates}

The procedure was approved and in accordance with the guideline set of the Laboratory Animal Care and Use Committee at the Medical College of Fudan University. A total of $2.8 \times 10^{5}$ i.u. purified RSV vitral particles were inactivated by heat at $65^{\circ} \mathrm{C}$ for $20 \mathrm{~min}$ and followed by formulation with $2 \mu \mathrm{g}$ ODN 1826, a synthetic oligodeoxynucleotide (Sigma) at room temperature. 10-12 week-old pregnant C57BL/B6 mice were nasal vaccinated with the $2.8 \times 10^{5}$ i.u. inactivated RSV at 2 weeks of gestation under anesthesia. The pregnant mice were vaccinated with $\mathrm{PBS}$ as negative controls. The same litter of offspring born to vaccinated and the same litter of offspring born to unvaccinated mother were intranasally immunized with $0.6 \times 10^{5}$ inactivated RSV plus CpG adjuvant in $10 \mu \mathrm{l}$ volume at the age of day 3 (designed as groups RSV-RSV and 0-RSV). In addition, the same litter of naïve neonates born to nasal vaccinated and unvaccinated mother were used as control groups (designed as groups RSV-0 and 0-0). All offspring were nasal challenged with live $2.8 \times 10^{5}$ i.u. RSV at 6 weeks old. Lung, spleen and blood were collected at the indicated time points.

\section{Anti-RSV antibody assay}

Serum anti-RSV IgG antibody in the treated mice was measured by ELISA assay. Briefly, serum was serial diluted and incubated in $1 \mu \mathrm{g} / \mathrm{ml}$ inactivated RSV-coated 96-well plate. After 24 hours, HRP-conjugated second antibody against mouse total IgG was added. The reaction was developed using TMB. In addition, a serial diluted commercial mouse anti-RSV IgG (Santa Cruz, CA, USA) was used as standard protein control to calculate serum anti-RSV antibody titer concentration. Data was presented as $\mathrm{mg} / \mathrm{ml}$.

In addition, serum anti-RSV neutralizing antibody was measured in Vero cells. Briefly, $1.4 \times 10^{5}$ i.u.live RSV was incubated with the treated mouse serum at the indicated dilution for 2 hours at $37^{\circ} \mathrm{C}$ for neutralization of RSV. $1 \times 10^{5}$ Vero cells were infected with the pre- treated RSV for 48 hours. The RSV infected cells were detected by immunostaining. The cells infected with RSV pre-mixed with naïve mouse serum were used as control. Data was presented as percentage of RSV titer reduction as compared to the naïve serum.

\section{IFN-gamma and IL-4 assay}

IFN-gamma and IL-4 levels in the supernatants of stimulated splenocytes were measured by ELISA assay. Briefly, $1 \times 10^{6}$ splenocytes from the treated offspring were stimulated or unstimulated with $1 \times$ $10^{4}$ i.u. inactivated RSV for 3 days. IFN-gamma and IL- 4 in the supernatants were measured by ELISA kit (R\&D system). Data was presented as $\mathrm{pg} / \mathrm{ml} \pm$ standard error. In addition, IFN-gamma and IL-4 production from the treated cells were detected by intracellular staining after 1 day of stimulation with $1 \times 10^{4}$ i.u. inactive RSV. 300 $\mathrm{ng} / \mathrm{ml}$ brefeldin A was added in the last 6 hours to prevent cytokine release. The cells were stained with antibodies against CD4 and CD44 (eBioscience), and followed by intracellular staining for IFN-gamma. Data was analyzed on Flow Jo software after the samples were run on BD FACSCanto II flow cytometry system.

\section{Statistical analysis}

Results are presented as mean \pm standard error (SE) of each group. Differences between multiple groups were evaluated by KruskalWallisone way ANOVA. Experiments with 2 groups were tested for statistical significance using unpaired, two-tailed Student's $t$ test. A value with less than 0.05 was considered as statistically significant.

\section{Results}

\section{RSV vaccination induced production of anti-RSV antibody}

In this study, we amplified and purified RSV viral particles. The heat-inactivated RSV was used for RSV vaccination. The pregnant mice were nasal vaccinated with $2.8 \times 10^{5}$ i.u. inactivated RSV at 2 weeks of post-gestation. Serum anti-RSV IgG was measured by ELISA assay. We observed a gradually increased total anti-RSV IgG at 2 week post-immunization and the antibodies continued to increase and remained at a stable level until 6 weeks (Figure 1A). The end-point anti-RSV titer reached up to 32000 at 4 weeks post-vaccination (Figure 1B). There is no sign of sickness during the observation period. The results indicated that the inactivated RSV has no side effects and can be safely used as vaccine antigen in animal model. To meaure RSV neutralizing antibody, $1.4 \times 10^{5}$ i.u. live RSV were pre-incubated with serum containing anti-RSV IgG from immunized mother to achieve RSV neutralization. Then Vero cells were infected with the neutralized RSV. After 24 hour infection, we observed that RSV titer was significantly reduced in the cells infected with RSV that was preincubated with mouse serum collected at 4 and 8 weeks postvaccination, indicating that serum anti-RSV antibody can effectively neutralize RSV (Figure 1C). Further analysis showed that serum antiRSV can effectively bind to RSV viral particles in the infected Vero cells; the plagues were positively stained with serum anti-RSV antibody titer after immunostaining (Figure 1D). In contrast, we did not observe positive RSV staining using naive serum. The results indicated that maternal RSV vaccination induced high of anti-RSV that can effectively reduce Vero cells from RSV infection. 


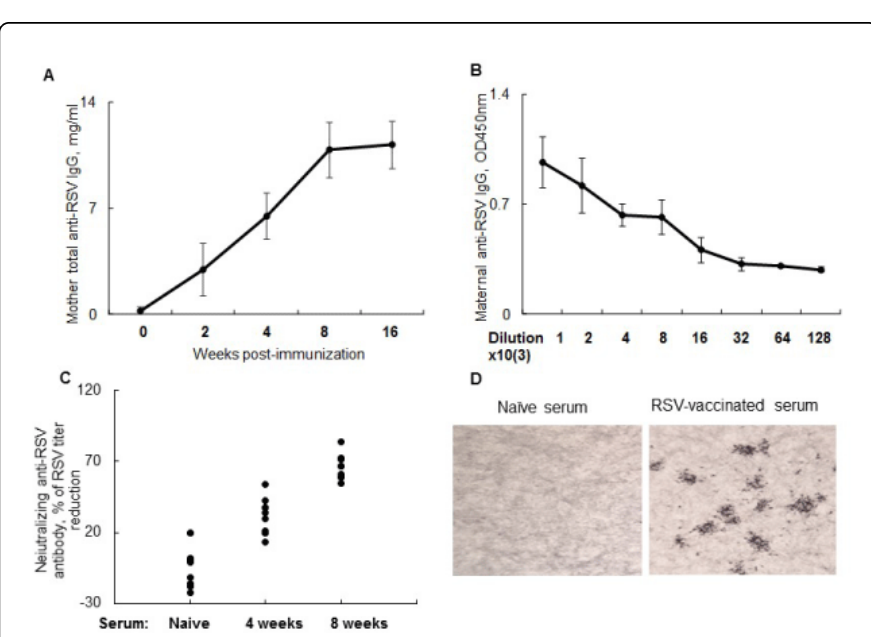

Figure 1: Maternal RSV vaccination induced production of serum anti-RSV antibodies. Pregnant mice were vaccinated via nasal injection of $2.8 \times 10^{5}$ i.u. of heat inactivated RSV at 2 weeks of postgestation. (A) Serum anti-RSV IgG was measured in mouse serum collected in different time points after vaccination. 96-well plate was coated with $1 \mathrm{mg} / \mathrm{ml}$ inactivated RSV and followed by incubation with serial diluted serum. A serial dilution of commercial mouse IgG was coated in 96-well plate as standard protein control. Data was presented as mean antibody concentration $(\mathrm{mg} / \mathrm{ml}) \pm$ standard error, $n=5$. One of at least 3 independent experiments was shown. (B) Total serum anti-RSV IgG was detected in mice 4 weeks postvaccination. Data was presented as mean OD450 $\mathrm{nm} \pm$ standard error, $n=5$. (C) Anti-RSV neutralizing antibody was analyzed by infection of Vero cells. $1.4 \times 10^{5} \mathrm{i} . \mathrm{u} / \mathrm{ml}$ RSV was pre-neutralized by incubation with RSV-vaccinated mouse serum (dilutuion 1:1) collected from mice 4 and 8 weeks post-vaccination. The same amount of RSV was pre-incubated with naïve mouse serum as control. The positive infected cells were detected by plague assay after 24 hours infection. Data was presented as percentage of reduction in virus titer relative to the naïve serum controls. Representative data of 2 independent experiments was shown. $\mathrm{n}=10$. (D) Anti-RSV IgG was identified in RSV-infected Vero cells. The infected cells were incubated with naïve serum or RSV vaccinated serum (4 weeks post-vaccination, dilution 1500). AntiRSV antibody was detected by HRP-conjugated anti-mouse IgG and developed by DAB. One of representative data was shown.

\section{Combinational passive and active vaccination reduced lung RSV load and inflammation after neonatal RSV challenge}

To determine the effects of combinational passive and active vaccination on protection against RSV infection and lung inflammation in neonatal mice, the pregnant mother mice were received nasal vaccination with inactivated RSV at day 14 of gestation and the same litter of offspring was nasal vaccinated with inactivated RSV at day 3 (Group RSV-RSV). The same litter of actively vaccinated neonates born to un-vaccinated mother (Group 0-RSV) and those naive neonates born to the immunized mother (Group RSV-0) and born to naïve mother (0-0) were used as control groups (Figure 2A). We observed no differences in growth, mortality and mobility of those neonates after vaccination during our experimental timepoints. To investigate the effects of RSV challenge on lung inflammation and virus load, the neonates among 4 groups was nasal challenged with 2.8 $\times 10^{5}$ i.u. RSV at 6 week-old. We observed a massive destruction of lung parenchyma and inflammatory infiltrates in the interstitial area and around bronchi of the lung tissues among groups 0-0 and 0-RSV 1 week after challenge. However the inflammatory infiltrates were greatly reduced in group RSV-RSV, in which offspring was born to immunized mother and received active immunization at day 3 after birth. There was moderate reduction of inflammatory infiltrates in the lung of naïve offspring born to immunized mother (group RSV-0). The offspring received active immunization in group 0 -RSV achieved the less protection than groups RSV-0 and RSV-RSV (Figure 2B and 2C).

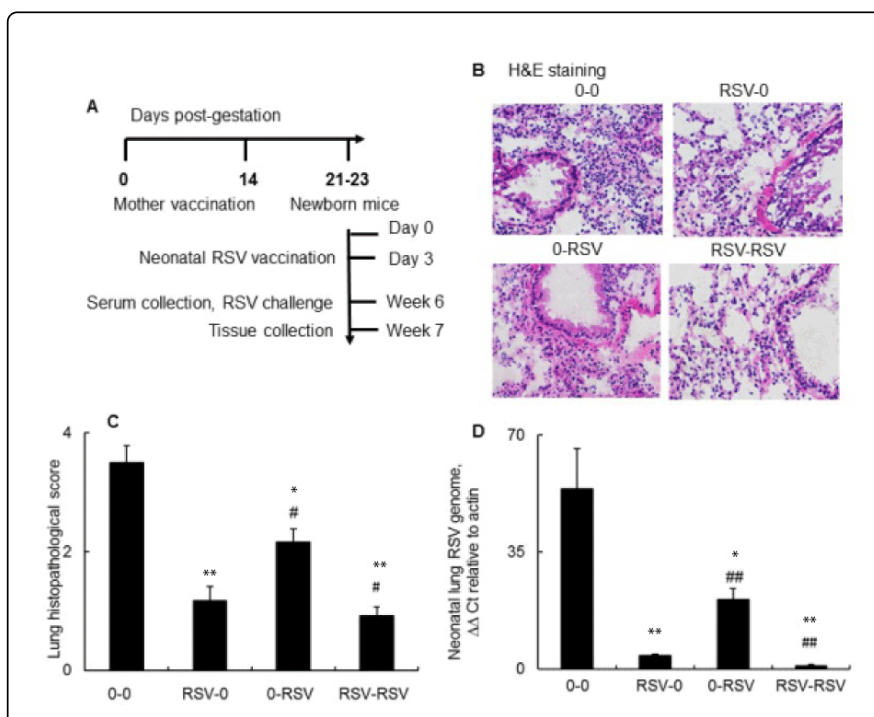

Figure 2: Combinational maternal and neonatal active vaccination reduced lung RSV load and inflammation after neonatal RSV challenge. (A) Schematic diagram of maternal and neonatal vaccination. After 14 days of gestation, mothers were intranasal vaccinated with $2.8 \times 10^{5}$ i.u. heat-inactivated RSV. The same litter of pups were intranasal vaccinated with $0.6 \times 10^{5}$ i.u RSV 3 days after birth. The same litter of unvaccinated mother and newborn pups were used as controls. The vaccinated offspring were challenged with $2.8 \times 10^{5}$ i.u. RSV at 6 weeks old. One week postRSV challenge, lung inflammation was analyzed by H\&E staining. The offspring in RSV-RSV group had the lowest lung inflammatory infiltrates. One representative photograph in each group was shown (B). Histology was scored for severity on scale of 0 to 4 in terms of infiltrating inflammatory cells. Data was presented as lung histological score (C). $n=5 .{ }^{* *} \mathrm{p}<0.01$ compared to group $0-0$, ${ }^{\#} \mathrm{p}<0.05,{ }^{\# \#} \mathrm{p}<0.01$ compared to group RSV-0. (D) Lung RSV genomes were analyzed by quantitative RT-PCR analysis 1 week after RSV challenge. Data is presented as mean $\Delta \Delta$ Ct relative to internal control actin \pm standard error. One representative data of 2 independent experiments was shown.

Further investigation on RSV genome by quantitative PCR revealed that RSV genomes largely existed in the challenged lung tissues of $0-0$ group (Figure 2D). However, RSV genomes were significantly attenuated in group RSV-0 and more greatly reduced in group RSVRSV than that of RSV- 0 group $(\mathrm{p}<0.01, \mathrm{n}=5)$, implying the protective role of maternal anti-RSV antibody in neonatal defense against RSV infection. In addition, we observed a moderately decreased RSV load in 0 -RSV group as compared to the control group $0-0 \quad(\mathrm{p}<0.05)$, 
suggesting that neonatal active vaccine alone (group 0-RSV) can effectively protect offspring from RSV infection to some extent. But the protective ability was significantly lower than those among groups RSV-0 and RSV-RSV. Therefore, combinational passive and active vaccination reduced lung RSV load and inflammation after neonatal RSV challenge.

\section{Combinational passive and active vaccination increased serum anti-RSV antibody}

To investigate whether the increased protection against RSV in neonates is associated with the enhanced humeral responses, we measured offspring serum total anti-RSV IgG antibody and IgG subclasses. Our results indicated that there were high levels of total anti-RSV IgGt in RSV-0 and RSV-RSV groups at the age of 6 weeks old. The anti-RSV IgGt was significantly higher in RSV-RSV group than that in RSV-0 group $(8.31 \pm 1.35 \mathrm{mg} / \mathrm{ml}$ vs. $2.53 \pm 0.76 \mathrm{mg} / \mathrm{ml})$ (Figure $3 \mathrm{~A}$ ), indicating that the offspring produced a high level of endogenous anti-RSV IgG in group RSV-RSV, in addition to the remaining amount of transferred maternal anti-RSV IgGt in offspring. However the antibody was undetectable in $0-0$ and low in $0-$ RSV groups. Thus active vaccination in neonates born to unimmunized mother was not effective, that was correlated to high RSV load in the infected lung tissues in the two groups. Similarly, anti-RSV subclass IgG1 was significantly increased in groups RSV-RSV and RSV-0, but almost undetectable in 0-0 and 0-RSV groups at 6 weeks old offspring. We did not detect markedly increased anti-RSV subclass IgG2a in groups RSV-RSV and RSV-0, but there was slightly and significantly increased IgG2a in group RSV-RSV as compared to RSV-0, however the change did not result in alteration of IgG1/IgG2a ratio in two groups (Figure 3B).
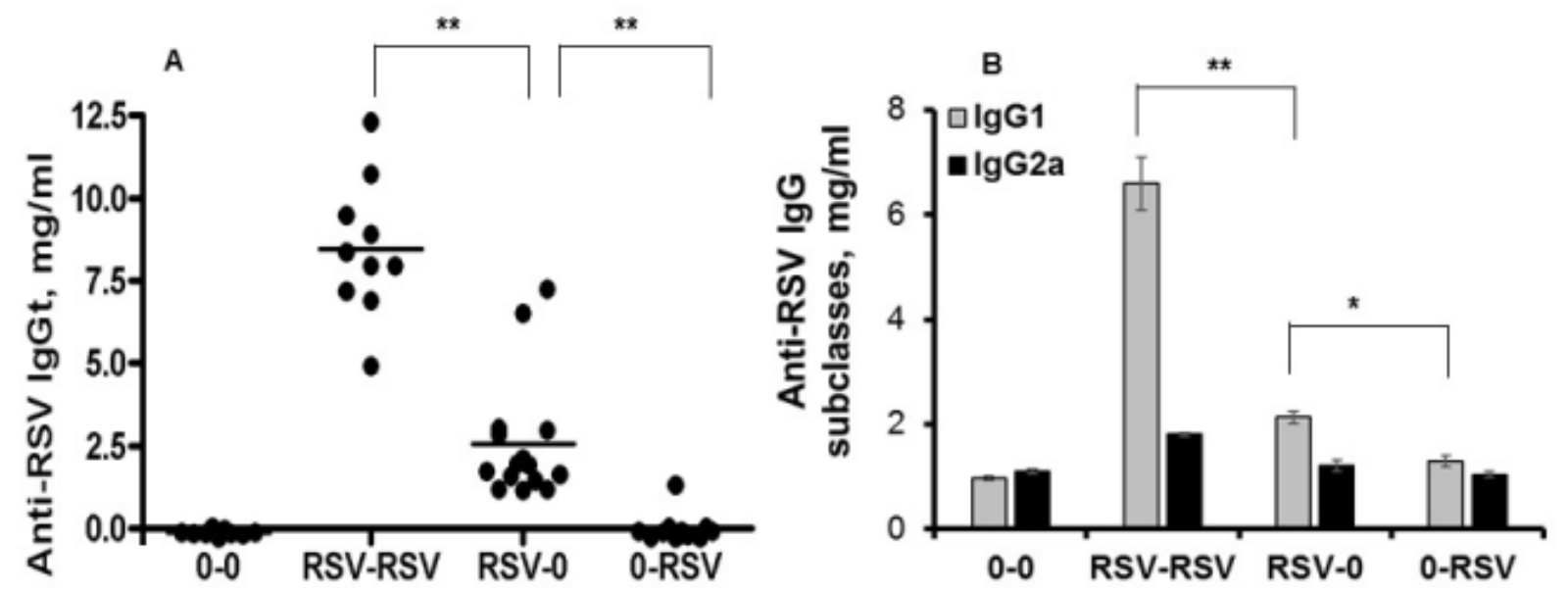

C

$0-0$

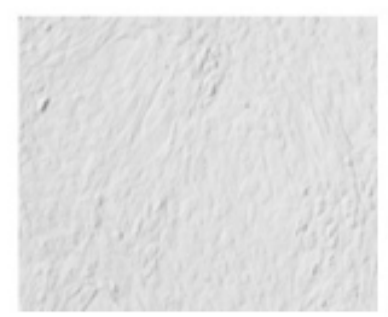

RSV-RSV

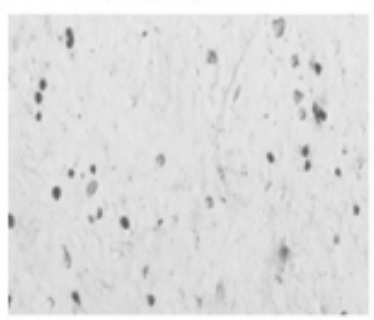

RSV-0

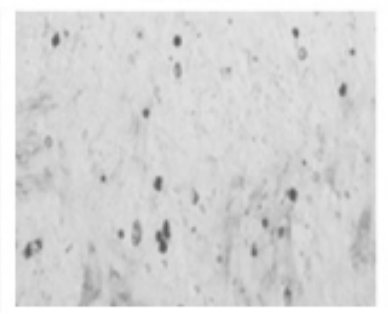

0-RSV

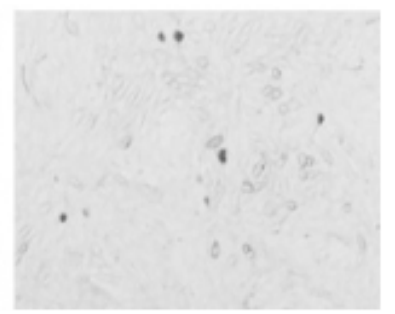

Figure 3: Combinational active maternal and neonatal vaccination increased serum anti-RSV antibody. Serum anti-RSV antibody was analyzed by ELISA assay. (A) Serum from 6 weeks old offspring was diluted by 1000-fold and incubated with the inactivated RSV antigen coated in 96-well plate overnight. A serial dilution of commercial mouse total IgG, IgG1 and IgG2a were coated in 96-well plate as standard protein controls. Anti-RSV total IgG was measured by incubation with HRP-conjugated anti-mouse total IgG. Data was presented as anti-RSV IgGt $\mathrm{mg} / \mathrm{ml}$. Pooled data of two independent experiments was shown. ${ }^{*} \mathrm{p}<0.05,{ }^{* *} \mathrm{p}<0.01$; (B) Anti-RSV subclass IgGs concentration in serum of offspring at 6 weeks old was measured by incubation with HRP-conjugated anti-mouse IgG1 or IgG2a. One representative data was shown. $\mathrm{n}=5,{ }^{*} \mathrm{p}<0.05,{ }^{* *} \mathrm{p}<0.01$. (C) Serum anti-RSV antibody specifically binds to RSV virus in the infected Vero cells. $5 \times 10^{5}$ Vero cells were infected with RSV at MOI=0.1 for 24 hours. Serum sample of individual mouse at 6 weeks old was diluted at 1:1000, then incubated with the RSVinfected cells and followed by HRP-conjugated anti-mouse second antibody. The stained cells were developed by DAB. One representative photograph in each group was shown.

Thus pre-existing maternal antibody did not interfere with, but improved endogenous production of anti-RSV IgG in the vaccinated neonates. Our additional study revealed that serum anti-RSV can effectively bind to the RSV infected cells, with the highest specific RSV binding activity of serum from RSV-RSV group that was correlated to the antibody titer results by ELISA assay (Figure 3C). 
Page 5 of 7

\section{Combinational passive and active vaccination increased IFN- gamma production from ex vivo stimulated splenocytes}

To investigate whether active neonatal RSV vaccination induced Th1-biased cellular immune responses under the presence of maternal antibody, we measured IFN-gamma and IL-4. Similarly we observed the significantly increased IFN-gamma expression that reached up to $514 \pm 238$ and $938 \pm 67 \mathrm{pg} / \mathrm{ml}$ in RSV-0 and RSV-RSV groups as compared to those in 0-0 control group $(181 \pm 24 \mathrm{pg} / \mathrm{ml})$ after restimulation with inactivated RSV ex vivo $(\mathrm{p}<0.05)$ (Figure 4A, upper panel).

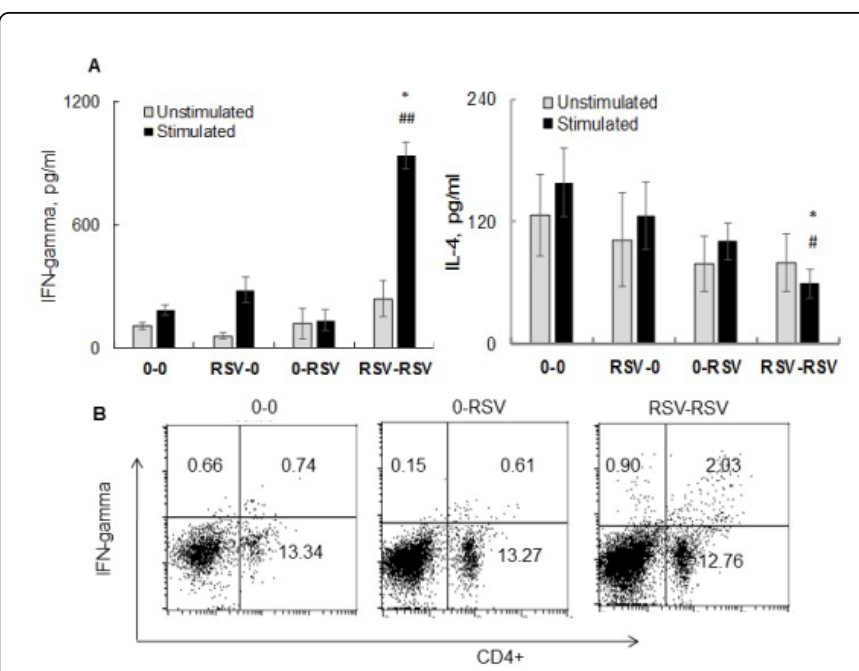

Figure 4: Combinational active maternal and neonatal vaccination increased IFN-gamma production from ex vivo stimulated splenocytes. (A) IFN-gamma and IL-4 were analyzed in the splenocytes from the vaccinated mice. $1 \times 10^{6}$ splenocytes were unstimulated or stimulated with inactivated $1 \times 10^{4}$ i.u. RSV for 3 days. The cell supernatants were collected for measurement of IFNgamma and IL-4. Data was presented as mean $\mathrm{pg} / \mathrm{ml} \pm$ standard error, $\mathrm{n}=5$. ${ }^{*} \mathrm{p}<0.05,{ }^{* *} \mathrm{p}<0.01$ compared to naïve pups born to unimmunized mother (0-0 group); ${ }^{\#} \mathrm{p}<0.05,{ }^{\# \#} \mathrm{p}<0.01$ compared to RSV-0 group. One representative data of 3 independent experiments was shown. (B) IFN-gamma expression from CD4+ T cells were analyzed by intracellular staining after 1 day of stimulation. Data was presented as dot plot. One representative data was shown.

However the active neonatal vaccination in 0-RSV group achieved moderate, but not significantly increased IFN-gamma 6 weeks after neonatal vaccination $(\mathrm{p}<0.05)$. In contrast, IL-4 expression was not increased, but moderately decreased among 0-RSV and RSV-RSV groups as compared to 0-0 group (Figure 4A, lower panel) ( $\mathrm{p}>0.05)$. The mean ratio of IFN-gamma/IL-4 was moderately increased for 1.3 and 1.6-fold in 0-RSV and RSV-RSV groups, suggesting that combinational vaccination synergistically increased the Th1-biased cellular immune responses. The results were further confirmed by intracellular staining, in which IFN-gamma production from $\mathrm{T}$ lymphocytes were more increased in in RSV-RSV than 0-0 and 0-RSV groups (Figure 4B), but we did not detect positive IL-4 expressing T lymphocytes in all three groups by intracellular staining (data not shown). IL-4 transgenic 4get mice expressing GFP under $I L-4$ gene promoter would improve detection limit of low IL-4 expression level in cells by intracellular staining. The results indicated that combinational vaccination greatly increased Th1-biased immune responses in vivo.

\section{RSV immune complex stimulated IFN-gamma expression from splenocytes in vitro}

To study the underlying immunological mechanisms of the increased Th1-biased cellular immune responses in the combinational vaccinated neonates, we performed ex vivo studies, in which splenocytes were stimulated in vitro with RSV immune complex. Immune complex was formed by incubation of inactivated RSV particles with RSV vaccinated serum which contained high concentration of anti-RSV IgG; whereas the inactivated RSV particles incubated with naïve serum without the presence of anti-RSV antibody or inactivated RSV alone were used as stimulation controls. We observed a moderately increased IFN-gamma production in the cells stimulated RSV immune complex, as compared to the cells stimulated with two controls, in which RSV was mixed with naïve serum or RSV alone (17.59\% vs. $11.65 \%$ and $10.67 \%)$ (Figure 5A).

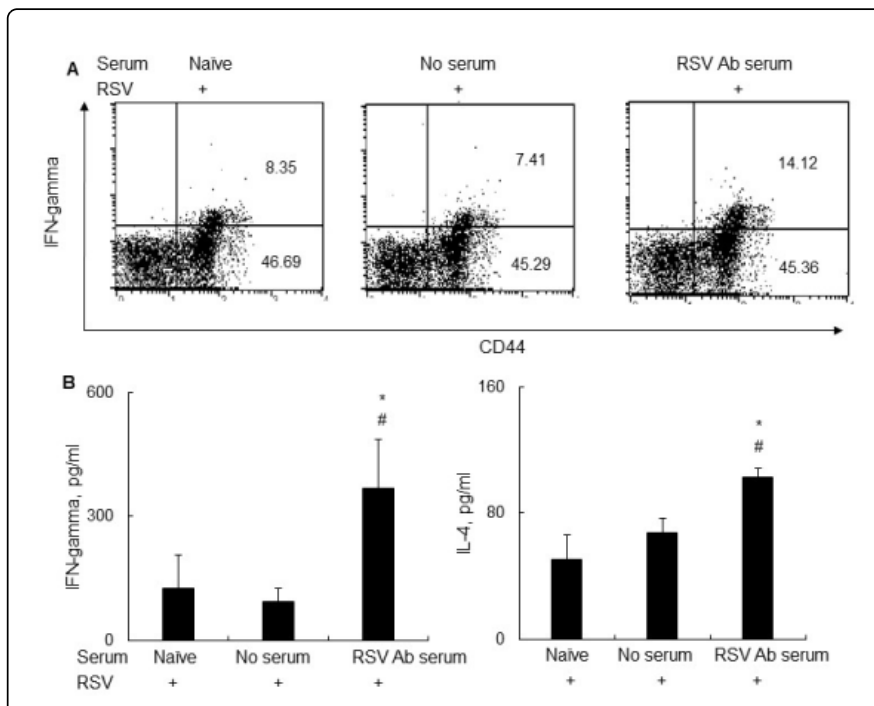

Figure 5: RSV immune complex stimulated production of IFNgamma and anti-RSV antibody from splenocytes in vitro. (A) $10 \mu \mathrm{l}$ mouse serum from the immunized mice was incubated with $1 \times 10^{5}$ i.u. inactivated RSV for 2 hours at $37^{\circ} \mathrm{C}$ to form immune complex. Naïve serum $(\mathrm{NV})$ was used as controls. $1 \times 10^{6}$ splenocytes from naïve mice were respectively stimulated for 3 days with immune complex (RSV Ab serum incubated with RSV). The cells stimulated with naive serum incubated with RSV or inactivated RSV alone was used as controls. IFN-gamma expression in the stimulated splenocytes was analyzed by intracellular staining. Data was presented as dot plot. One representative data of 2 independent experiments was shown. (B) IFN-gamma and IL-4 in conditioned medium of the stimulated splenocytes were measured by ELISA. Data was presented as mean \pm standard error, $\mathrm{n}=4 .{ }^{*} \mathrm{p}<0.05,{ }^{* *} \mathrm{p}<0.01$ compared to the naïve serum incubated RSV-treated cells, ${ }^{\#} \mathrm{p}<0.05$, ${ }^{\# \#} \mathrm{p}<0.01$ compared to the RSV alone-stimulated splenocytes.

IL-4 expression was very low and undetectable among three treatments (data not shown). Further analysis indicated that the increased IFN-gamma was exclusively produced from CD44+ memory $\mathrm{T}$ cells (Figure 5A). The results were further confirmed by ELISA assay, 
in which RSV immune complex stimulation moderately increased IFN-gamma expression as compared to the cells stimulated with RSV mixed with naïve serum or RSV alone $(366.8 \pm 120.5 \mathrm{pg} / \mathrm{ml} v s .127 .8 \pm$ 79.3 and $94.4 \pm 32.9 \mathrm{pg} / \mathrm{ml})(\mathrm{p}<0.05)$ (Figure $5 \mathrm{~B})$. Though IL-4 was slightly increased in RSV immune complex stimulated cells, the ratio of IFN-gamma/IL-4 was significantly increased in the immune complex-stimulated cells, indicating that immune complex or maternal anti-IgG antibody promoted Th1 biased $\mathrm{T}$ cell polarization (data not shown). The results indicated that RSV immune complex can improve production of IFN-gamma and skew Th1 biased T cell polarization.

\section{Discussion}

In this study, we explored a novel neonatal vaccination strategy, in which neonates were actively vaccinated with inactivated RSV particles under the presence of maternal anti-RSV antibody. Our results revealed that active neonatal RSV vaccination born to RSV immunized mother improved immune protection against RSV infection than maternal passive or neonatal active immunization alone. Active neonatal vaccination alone provided low immune protection following RSV challenge. The beneficial effects were in association with higher production of anti-RSV antibody and IFN-gamma in neonates received the combinational immunization. In contrast, low protection in 0-RSV group was correlated with low and undetectable level of antiRSV IgG. It is known that IgG1 and IgG2a respectively represent allergic Th2 and immune protective Th1 immune responses. IgG2a isotype has strong neutralization activity [17]. We observed a comparable ratio of IgG1/IgG2a in RSV-RSV and RSV-0 group, demonstrating that actively vaccinated offspring born to RSV immunized mother achieved a similar balanced Th1/Th2 immune response as their mother. Maternal anti-RSV IgG in offspring that was not received active immunization moderately diminished at 6 weeks, whereas anti-RSV IgG level remained at relatively stable level for up to 6 weeks among offpring received combinational immunization (Figure $3 \mathrm{~A}$ ). The results implyed the endogenous production of anti-RSV by neonatal immune system, in consistent with the previous report, in which neonates were vaccinated two times at 1 and 3 weeks old with RSV protein Gcf under the presence of pre-preexisting maternal antibody and achieved stable endogenous anti-RSV IgG level for up to 8 weeks [13]. Thus immunization with inactivated RSV under the presence of maternal anti-RSV IgG can induce long-lasting systemic immune protection. In addition, our results also showed that neonates can be immunized as early as day 3 after birth to achieve comparable immune protection as immunized at 1 and 3 weeks old.

In consistent with the increased systemic immune responses, we observed the suppressed lung inflammation after live RSV challenge. The inflammatory infiltrates were significantly attenuated in offspring received combinational vaccination as compared to the neonates received active vaccination alone. The results support the beneficial role of combinational vaccination in overcoming Th2 type biased immune responses that was induced by neonatal FI RSV or $\mathrm{Gcf}^{+} \mathrm{CT}$ vaccination. Early active vaccination with crude inactive RSV at day 3 after birth can increase Th1-biased cytokine production and the results cannot be achieved when offspring was received vaccination for two times at 1 and 3 weeks old with RSV protein Gcf [13].The suppressed lung inflammation was associated with significant lower lung RSV load after RSV challenge. RSV viral genomes were detected in $10 \%$ lung tissues of group RSV-RSV after RSV challenge, but the percentage was much higher in groups RSV-0, 0 -RSV and 0-0 that reached for 30\%, $33.3 \%$ and $95 \%$ respectively at 1 week post-RSV challenge (data not shown). The results further indicated that combinational passive and active vaccination provided better protection against RSV infection than passive and active vaccination alone. The beneficial effects were associated with elevated production of anti-RSV IgG and improved Th1-biased $\mathrm{T}$ cell polarization. Maternal antibody did not interfere with, but enhanced systemic immune responses in offspring, in agreement with previous reports in mouse and lamb animal models $[12,13]$.

To further investigate the underlying mechanisms of maternal antiRSV in enhancing neonatal active immunization, we further performed in vitro study, in which splenocytes were stimulated with RSV immune complex. As a result, we observed a significantly greater activation of splenocytes as compared to the cells stimulated with RSV antigen alone. Memory T cells are identified as CD44+CD62L- cell subset. After stimulation with RSV immune complex, we observed an increase in IFN-gamma from CD44+ $\mathrm{T}$ cells, but IFN-gamma expression level was low or undetectable in the unstimulated and RSV particle alone stimulated splenocytes, indicating that RSV immune complex acounted for the increased Th1-biased immune responses in vitro and in vivo. We expect that immune complex-mediated vaccination in neonates may induce a long-term protection against RSV infection through enhanced priming of memory $\mathrm{T}$ cells and production of IFN-gamma. It warrants us to further investigate the beneficial effects of immune complex in neonatal vaccination by direct nasal vaccination with immune complex.

We speculated that the increased antigen binding and up-take of immune complex by antigen presenting cells (APCs) may account for the increased Th1 immune responses. Because antibody Fc receptors are highly presented on the surface of mucosal APCs. Theoretically, APCs may internalize RSV immune complex more efficiently than RSV antigen alone. A body of evidences shows that antibody immune complex is able to increase antigen up-take and improve immune responses. For example, FcRn transgenic mice can produce greater amount of virus-specific antibody than wild-type mice after OVA immunization. The effects are associated with the increased phagocytosis of IgG immune complex [18]. In addition, vaccination with chimeric proteins composed of HIV Gag (p24) fused to Fc region of IgG induced more local and systematic immunity than antigen that was not infused with IgG Fc region [19]. However, mice lack of Fc receptor in Fc gamma RIII knock-out mice had impaired binding and phagocytosis of IgG1-coated erythrocytes by macrophages [20]. Therefore, we conclude that combinational RSV vaccination in neonates improved systemic and Th1-biased immunity possibly through the enhanced APCs antigen up-take and internalization at mucosal site after formation of RSV immune complex.

It is considered previously that neutralizing antibody blocks antigen internalization or phagocytosis of pathogens by target cells. For example, pre-existing maternal antibody interferes with neonatal active vaccination with Sendai virus envelop protein, but the suppressive effects were diminished after adulthood [21]. The similar effects are also demonstrated in viral vector-mediated gene therapy, because preexisting anti-adenoviral vector neutralizing antibody in vivo can diminish vector-mediated gene transfer into target cells and cause transient therapeutic gene expression, but the adverse effects can be reversed by immune suppressants and depletion of immune cells $[22,23]$. It is unknown how the neutralizing antibody has distinct effects on different animal models. Further study is warranted to investigate the underlying molecular and immunological mechanisms 
of anti-RSV maternal antibodies in modulation of neonatal immune system.

Taken together, maternal antibody can be used as potent adjuvant in RSV neonatal vaccination. Pre-existing maternal antibody induced long-term and potent Th1-biased neonatal immune responses against RSV in conjunction with active immunization. RSV immune complex is presented as a potent mediator in modulation of immune responses in neonatal vaccination.

\section{Acknowledgements}

This study was supported by research grant from Zhongshan Hospital, Fudan University in China to Z.J. (A654116001).

\section{Disclosure}

The authors declare no competing financial interests. None of the authors affiliated with this manuscript have any commercial or associations that might pose a conflict interest.

\section{Declaration of Interest}

The authors report no conflicts of interest. The authors alone are responsible for the content and writing of the paper.

\section{References}

1. Lambert L, Sagfors AM, Openshaw PJ, Culley FJ (2014) Immunity to RSV in Early-Life. Front Immunol 5: 466.

2. Zhang Y, Yuan L, Zhang Y, Zhang X, Zheng M, et al. (2015) Burden of respiratory syncytial virus infections in China: Systematic review, metaanalysis. Jogh 5: 020417.

3. Gervassi AL, Horton H (2014) Is Infant Immunity Actively Suppressed or Immature? Virology : research, treatment 4: 1-9.

4. Diesner SC, Forster-Waldl E, Olivera A, Pollak A, Jensen-Jarolim E, et al. (2012) Perspectives on immunomodulation early in life. Pediatric allergy, immunology: official publication of the European Society of Pediatric Allergy, Immunology 23: 210-223.

5. Jiang J, Fisher EM, Murasko DM (2013) Intrinsic defects in CD8 T cells with aging contribute to impaired primary antiviral responses. Experimental gerontology 48: 579-586.

6. Jiang J, Fisher E, Murasko DM (2013) Impaired specific CD8 T cell response with aging is not due to decreased expression of CD90 on TCR transgenic $\mathrm{T}$ cells. Immunity \& ageing 10: 36 .

7. You D, Siefker DT, Shrestha B, Saravia J, Cormier SA (2015) Building a better neonatal mouse model to understand infant respiratory syncytial virus disease. Respiratory research 16: 91.

8. Tregoning JS, Wang BL, McDonald JU, Yamaguchi Y, Harker JA, et al. (2013) Neonatal antibody responses are attenuated by interferon-gamma produced by NK, T cells during RSV infection. Proceedings of the National Academy of Sciences of the United States of America 110: 5576-5581.
9. Venkatesh MP, Weisman LE (2006) Prevention, treatment of respiratory syncytial virus infection in infants: an update. Expert Rev Vaccines 5: 261-268.

10. Glenn GM, Fries LF, Smith G, Kpamegan, Lu E, et al. (2015) Modeling maternal fetal RSV $\mathrm{F}$ vaccine induced antibody transfer in guinea pigs. Vaccine 33: 6488-6492.

11. Glenn GM, Fries LF, Thomas DN, Smith G, Kpamegan E, et al. (2016) A Randomized, Blinded, Controlled, Dose-Ranging Study of a Respiratory Syncytial Virus Recombinant Fusion (F) Nanoparticle Vaccine in Healthy Women of Childbearing Age. J Infec Diseases 213: 411-422.

12. Garg R, Latimer L, Gerdts V, Potter A, Hurk VDLVDS (2015) The respiratory syncytial virus fusion protein formulated with a novel combination adjuvant induces balanced immune responses in lambs with maternal antibodies. Vaccine 33: 1338-1344.

13. Noh Y, Shim BS, Cheon IS, Rho S, Kim HJ, et al. (2013) Neonatal immunization with respiratory syncytial virus glycoprotein fragment induces protective immunity in the presence of maternal antibodies in mice. Viral immunology 26: 268-276.

14. Morris MC, Surendran N (2016) Neonatal Vaccination: Challenges, Intervention Strategies. Neonatology 109: 161-169.

15. Remot A, Roux X, Dubuquoy C, Fix J, Bouet S, et al. (2012) Nucleoprotein nanostructures combined with adjuvants adapted to the neonatal immune context: a candidate mucosal RSV vaccine. PloS one 7 : e37722.

16. Jiang Z, Fehrenbach ML, Ravaioli G, Kokalari B, Redai IG, et al (2012) The effect of lipoprotein-associated phospholipase A2 deficiency on pulmonary allergic responses in Aspergillus fumigatus sensitized mice. Respiratory research 13: 100 .

17. Gros L, Pelegrin M, Michaud HA, Bianco S, Hernandez J, et al. (2007) Endogenous cytotoxic T-cell response contributes to the long-term antiretroviral protection induced by a short period of antibody-based immunotherapy of neonatally infected mice. J Virol 82: 1339-1349.

18. Cervenak J, Bender B, Schneider Z, Magna M, Carstea BV, et al. (2011) Neonatal FcR overexpression boosts humoral immune response in transgenic mice. J Immunol 186: 959-968.

19. Lu L, Palaniyandi S, Zeng R, Bai Y, Liu X, et al. (2011) A neonatal Fc receptor-targeted mucosal vaccine strategy effectively induces HIV-1 antigen-specific immunity to genital infection. J Virol 85: 10542-10553.

20. Hazenbos WL, Heijnen IA, Meyer D, Hofhuis FM, Lavalette RD, et al. (1998) Murine IgG1 complexes trigger immune effector functions predominantly via Fc gamma RIII (CD16). J Immunol 161: 3026-3032.

21. Blomqvist GA, Lovgren-Bengtsson K, Morein B (2003) Influence of maternal immunity on antibody, T-cell response in mice. Vaccine 21 : 2022-2031

22. Cao H, Wu J, Duan C, Du K, Lee CM, et al. (2016) Long-Term Expression of the Human CFTR Gene in Mouse Airway via Helper-Dependent Adenoviral Vector Delivery, Transient Immunosuppression. Human gene therapy 27: 83-9.

23. Alzuguren P, Hervas-Stubbs S, Gonzalez-Aseguinolaza G, Poutou J, Fortes P, et al. (2015) Transient depletion of specific immune cell populations to improve adenovirus-mediated transgene expression in the liver. Liver international: official journal of the International Association for the Study of the Liver 35: 1274-1289. 